\title{
Strong reflection principles and large cardinal axioms
}

\author{
J. Foukzon', E. R. Men'kova ${ }^{2}$ \\ ${ }^{1}$ Israel Institute of Technology, Haifa, Israel \\ ${ }^{2}$ LomonosovMoscowStateUniversity, Moscow, Russia
}

\section{Email address:}

E_Menkova@mail.ru(E. R. Men’kova),jaykovfoukzon@list.ru(J. Foukzon)

\section{To cite this article:}

J. Foukzon, E. R. Men'kova. Strong Reflection Principles and Large Cardinal Axioms. Pure and Applied Mathematics Journal. Vol. 2 , No. 3, 2013, pp. 119-127. doi: 10.11648/j.pamj.20130203.12

\begin{abstract}
In this article an possible generalization of the Löb's theorem is considered. We proved so-called uniform strong reflection principle corresponding to formal theories which has $\omega$-models.Main result is: let $\kappa$ be an inaccessible cardinal and $H_{\kappa}$ is a set of all sets having hereditary size less than $\kappa$, then: $\neg \operatorname{Con}\left(\mathrm{ZFC}+\left(\mathrm{V}=\mathrm{H}_{\kappa}\right)\right)$.
\end{abstract}

Keywords: Löb'stheorem, Second Gödelincompleteness Theorem, Consistency, Formal System, Uniform Reflection Principles, $\Omega$-Model Of $Z F C$, Standard Model Of $Z F C$, Inaccessible Cardinal, Weakly Compact Cardinal

\section{Introduction}

Let Th be some fixed, but unspecified, consistent formal theory.

Theorem1.[1].(Löb's Theorem).

If $T h \vdash \exists x \operatorname{Prov}_{T h}(x, \bar{n}) \rightarrow \Phi_{n}$ where $\mathrm{x}$ is the Gödel number of the proof of the formula with Gödel number $n$, and $\bar{n}$ is the numeral of the Gödel number of the formula $\Phi_{n}$ thenTh $\vdash \Phi_{n}$. Taking into account the second Gödelincompleteness theorem it is easyto see that $\Phi_{n}$ not be able to $\operatorname{prove} x \operatorname{Prov}_{T h}(x, \bar{n}) \rightarrow \Phi_{n}$, for disprovable (refutable) and undecidable formulas $\Phi_{n}$ Thus Löb's theorem says that for refutable or undecidable formulas $\varphi_{n}$, the intuition "if there is exists proof of $\Phi_{n}$ [i.e.Th $\left.\vdash \exists x \operatorname{Prov}_{T h_{\omega}}(x, \bar{n})\right]$ then $\Phi_{n}$ [i.e. $\left.\mathrm{Th} \vdash \Phi_{n}\right]$ " is fails. The reason of this phenomenon, consist in thatthe concept of natural numbers is not absolute and therefore in general case statement $T h \vdash \exists x \operatorname{Prov}_{T h_{\omega}}(x, \bar{n})$ does not asserts that: $T h \vdash \Phi_{n}$.

Definition 1.Let $M_{\omega}^{T h}$. be an $\omega$-model of the Th. We said that, Th\#is a nice theory over Thor a nice extension of the Thiff:

(i) $T h^{\#}$ contains $T h$;

(ii) Let $\Phi$ be any closed formula, then

$$
\left[T h \vdash \operatorname{Pr}_{T h}\left([\Phi]^{c}\right)\right] \wedge\left[M_{\omega}^{T h} \vDash \Phi\right]
$$

implies. , $T h^{\#} \vdash \Phi$.Here $[\Phi]^{c}$ is a code of $\Phi[2]$.

Definition2.We said that, Th\#is a maximally nice theory over Th or a maximally nice extension of the ThiffTh\#is consistent and for any consistent nice extension $T h^{\prime}$ of the Th: $\operatorname{Ded}\left(T h^{\#}\right) \subseteq \operatorname{Ded}\left(T h^{\prime}\right) \quad$ implies:. $\operatorname{Ded}\left(T h^{\#}\right)=$
$\operatorname{Ded}\left(T h^{\prime}\right)$.

Theorem2.(Generalized Löb's Theorem).Assume that (i) Con(Th) and (ii) Thhas an $\omega_{-}$-model $M_{\omega}^{T h}$. Then theoryThcan be extended to a maximally consistent nice theory Th\#.

Theorem3.(Strong Reflection Principle corresponding to $\omega$-model) Assume that (i) $\operatorname{Con}(T h)$, (ii) Th has an $\omega$-model $M_{\omega}^{T h}$.Let $\Phi$ be a Th-sentence and let $\Phi_{\omega}$ be a Th-sentence $\Phi$ relativized to a model $M_{\omega}^{T h}$.Then

$$
\begin{aligned}
& T h_{\omega} \vdash \Phi_{\omega} \leftrightarrow T h_{\omega} \vdash \operatorname{Pr}_{T h_{\omega}}\left(\left[\Phi_{\omega}\right]^{c}\right), \\
& T h_{\omega} \vDash \Phi_{\omega} \leftrightarrow T h_{\omega} \vDash \operatorname{Pr}_{T h_{\omega}}\left(\left[\Phi_{\omega}\right]^{c}\right) .
\end{aligned}
$$

Theorem4.Let $\kappa$ be an inaccessible cardinal.Then $\neg \operatorname{Con}(\mathrm{ZFC}+\exists \kappa)$.

Theorem5. $\neg$ Con(NFUA).

Theorem6. $\neg$ Con(NFUB).

\section{Preliminaries}

Let Thbe some fixed, but unspecified, consistent formal theory. For later convenience, we assume that the encoding is done in some fixed formal theory $S$ and that Thcontains $S$. We do not specify $S$--- it is usually taken to be a formal system of arithmetic, although a weak set theory is often more convenient. The sense in which $S$ is contained in $T h$ is better exemplified than explained: If $S$ is a formal system of arithmetic and This, say, $Z F C$, then $T h$ contains $S$ in the sense that there is a well-known embedding, or interpretation, of $S$ inTh. Since encoding is to take place in $S$, it will have to have a large supply of constants and closed terms to 
be used as codes (e. g. in formal arithmetic, one has $\overline{0}, \overline{1}, \ldots)$.S Will also have certain function symbols to be described shortly. To each formula $\Phi$ of the language of $T h$ is assigned a closed term $[\Phi]^{c}$ called the code of $\Phi$. If $\Phi$ is a formula with a free variable $x$, then $[\Phi(x)]^{c}$ is a closed term encoding the formula $\Phi(x)$ with $x$ viewed as a syntactic object and not as a parameter. Corresponding to the logical connectives and quantifiers are function symbols: $n \operatorname{eg}(\cdot), \operatorname{imp}(\cdot)$, etc., such that, for all formulae $\Phi, \Psi: S \vdash$ $\operatorname{neg}\left([\Phi]^{c}\right)=[\neg \Phi]^{c}, S \vdash \operatorname{imp}(\cdot)=[\Phi \rightarrow \Psi]^{c}$, etc. Of particular importance is the substitution operator, represented by the function symbolub(,$\cdot)$. For formulae $\Phi(x)$, terms $t$ with codes $[t]^{c}$ :

$$
S \vdash \operatorname{sub}\left([\Phi(x)]^{c},[t]^{c}\right)=[\Phi(t)]^{c} .
$$

Iteration of the substitution operator sub allows one to define function symbols $s u b_{1}, s u b_{2}, \ldots, s u b_{n}$, such that

$$
\begin{gathered}
S \vdash \operatorname{sub}_{n}\left(\left[\Phi\left(x_{1}, x_{2}, \ldots, x_{n},\right)\right]^{c},\left[t_{1}\right]^{c},\left[t_{2}\right]^{c}, \ldots,\left[t_{n}\right]^{c},\right)= \\
=\left[\Phi\left(t_{1}, t_{2}, \ldots, t_{n}\right)\right] .
\end{gathered}
$$

It well known [2],[3] that one can also encode derivations and have a binary relation $\operatorname{Prov}_{T h}(x, y)$ (read "x proves y" or " $\mathrm{x}$ is a proof of $\mathrm{y}$ ") such that for closed $t_{1}, t_{2}: S \vdash$ $\operatorname{Prov}_{T h}\left(t_{1}, t_{2}\right)$ ifft $t_{1}$ is the code of a derivation in Th of the formula with code $t_{2}$. It follows that

$$
T h \vdash \Phi \leftrightarrow S \vdash \operatorname{Prov}_{T h}\left(t,[\Phi]^{c}\right)
$$

for some closed term $t$. Thus one can define predicate $\operatorname{Pr}_{T h}(y)$ :

$$
\operatorname{Pr}_{T h}(y) \leftrightarrow \exists x \operatorname{Prov}_{T h}(x, y)
$$

and therefore one obtain a predicate asserting provability.

\section{Remark2.1.}

We note that is not always the case that [2]-[3]:

$$
T h \vdash \Phi \leftrightarrow S \vdash \operatorname{Pr}_{T h}\left([\Phi]^{c}\right) .
$$

It well known [3] that the above encoding can be carried out in such a way that the following important conditions D1,D2 and D3 are met for allsentences [2],[3]:

$$
\begin{gathered}
\text { D1. Th } \vdash \Phi \rightarrow S \vdash \operatorname{Pr}_{T h}\left([\Phi]^{c}\right), \\
\text { D2. } \operatorname{Pr}_{T h}\left([\Phi]^{c}\right) \rightarrow \operatorname{Pr}_{T h}\left(\left[\operatorname{Pr}_{T h}\left([\Phi]^{c}\right)\right]^{c}\right),(2.6) \\
\text { D3. } \operatorname{Pr}_{T h}\left([\Phi]^{c}\right) \wedge \operatorname{Pr}_{T h}\left([\Phi \rightarrow \Psi]^{c}\right) \rightarrow \operatorname{Pr}_{T h}\left([\Psi]^{c}\right) .
\end{gathered}
$$

Conditions D1,D2 and D3 are called the Derivability Conditions.

\section{Assumption2.1.}

We assume throughout that:

(i) the language of $T h$ consists of: numerals $\overline{0}, \overline{1}, \ldots$ ) countableset of the numerical variables: $\left\{v_{0}, v_{1}, \ldots\right\}$ countable set $F$ of the set variables:

$$
F=\{x, y, z, X, Y, Z, \mathfrak{I}, \mathfrak{K}, \mathfrak{M}, \mathfrak{N}, \ldots\} ;
$$

countable set of the $n$-ary function symbols: $f_{0}^{n}, f_{1}^{n}, \ldots, f_{m}^{n}, \ldots$; countable set of the $n$-ary relation bols: $R_{0}^{n}, R_{1}^{n}, \ldots, R_{m}^{n}, \ldots$;

connectives: $\neg, \rightarrow$;

quantifier: $\forall$.

(ii) $T h$ contains a theory $T h^{*}$ :

$$
T h^{*}=Z F C+\exists(\omega-\text { model of } Z F C) .
$$

(iii) Thhas an $\omega$-model $M_{\omega}^{T h}$.

\section{Theorem 2.1.}

(Löb’s Theorem). Let be (1) Con(Th) and (2) $\Phi$ be closed. Then

$$
T h \vdash \operatorname{Pr}_{T h}\left([\Phi]^{c}\right) \rightarrow \Phi .
$$

It well known that replacing the induction scheme in Peano arithmetic $P A$ by the $\omega$-rule with the meaning "if the formula $A(n)$ is provable for all $n$, then the formula $A(x)$ is provable" :

$$
\frac{A(0), A(1), \ldots, A(n)}{\forall x A(x)}
$$

leads to complete and sound system $P A_{\infty}$ where each true arithmetical statement is provable. S.Feferman showed that an equivalent formal system $T h^{\#}$ can be obtained by erecting on $T h=P A$ a transfinite progression of formal tems $P A_{\alpha}$ according to the following scheme

$$
\begin{gathered}
P A_{0}=P A \\
P A_{\alpha+1}=P A_{\alpha}+\left\{\forall x P_{P A_{\alpha}}\left([A(\dot{x})]^{c}\right) \rightarrow \forall x A(x)\right\} \\
P A_{\lambda}=\bigcup_{\alpha<\lambda} P A_{0},
\end{gathered}
$$

where $A(x)$ is a formula with one free variable and where $\lambda$ is a limit ordinal. Then $T h^{\#}=\bigcup_{\alpha \in O} P A_{0}$, Obeing Kleene's system of ordinal notations, is equivalentto a theory:. $P A_{\infty}$ It is easy to see that a theoryTh $h^{\#}=P A^{\#}=P A_{\infty}$, i.e. $T h^{\#} T h^{\#}$ is a maximally nice extension of thePA.

Generalized Löb's Theorem. Strong Reflection Principle Corresponding to $\omega$-model.

\section{Definition3.1.}

AnTh-wff $\Phi$ )(well-formed formula

$\Phi)$ is closed i.e., $\Phi)$ is a Th- sentence iff it has no free variables; a wff $\Psi$ is open if it has free variables. We'll use the slang ' $\mathrm{k}$ - place open wff' to mean a wff with $\mathrm{k}$ distinct free variables. Given a model $M^{T h}$ of the Thand a Thsentence $\Phi$, we assume known the meaning of $M^{T h} \vDash \Phi$ - i. e. $\Phi$ is true in $M^{T h}$, (see for example [4],[5],[6]).

\section{Definition3.2.}

Let $M \stackrel{T h}{\omega}$ be an $\omega$-model of the Th. We said that, $T h^{\#}$ is a 
nice theory over Th or a nice extension of the Thiff:

(i) $T h^{\#}$ contains a theory $\mathrm{Th}$;

(ii) Let $\Phi$ be any closed formula, then

$$
\left[T h \vdash \operatorname{Pr}_{T h}\left([\Phi]^{c}\right)\right] \wedge[M \quad \stackrel{T h}{\omega} \vDash \Phi]
$$

impliesthat: $T h^{\#} \vdash \Phi$.

\section{Definition3.3.}

We said that $T h^{\#}$ is a maximally nice theory over Thor a maximally nice extension of the ThiffT $h^{\#}$ is consistent and for any consistent nice extension $T h^{\prime}$ of the $T h$ : $\operatorname{Ded}\left(T h^{\#}\right) \subseteq$ $\operatorname{Ded}\left(T h^{\prime}\right)$ impliesDed $\left(T h^{\#}\right)=\operatorname{Ded}\left(T h^{\prime}\right)$.

Lemma3.1. Assume that: (i)Con(Th)and (ii) Th $\operatorname{Pr}_{T h}\left([\Phi]^{c}\right)$, where $\Phi$ is a closed formula. Then: Th $\forall$ $\operatorname{Pr}_{T h}\left([\neg \Phi]^{c}\right)$.

Proof.Let $\operatorname{Con}_{T h}(\Phi)$ be the formula

$$
\begin{gathered}
\operatorname{Con}_{T h}(\Phi) \triangleq \\
\forall t_{1} \forall t_{2} \neg\left[\operatorname{Prov}_{T h}\left(t_{1},[\Phi]^{c}\right) \wedge \operatorname{Prov}_{T h}\left(t_{2}, \operatorname{neg}\left([\Phi]^{c}\right)\right)\right] \\
\leftrightarrow \\
\neg \exists t_{1} \neg \exists t_{2}\left[\operatorname{Prov}_{T h}\left(t_{1},[\Phi]^{c}\right) \wedge \operatorname{Prov}_{T h}\left(t_{2}, \operatorname{neg}\left([\Phi]^{c}\right)\right)\right]
\end{gathered}
$$

where $t_{1}, t_{2}$ is a closed term. We note that under canonicalobservation, one obtain

$T h+\operatorname{Con}(T h) \vdash \operatorname{Con}_{T h}(\Phi)$ for any closedwff $\Phi$.

Suppose that: $T h \vdash P r_{T h}\left([\neg \Phi]^{c}\right)$, then assumption (ii) gives

$$
T h \vdash \operatorname{Pr}_{T h}\left([\Phi]^{c}\right) \wedge \operatorname{Pr}_{T h}\left([\neg \Phi]^{c}\right)
$$

From (3.1) and (3.2) one obtain(3.3)

$$
\exists t_{1} \neg \exists t_{2}\left[\operatorname{Prov}_{T h}\left(t_{1},[\Phi]^{c}\right) \wedge \operatorname{Prov}_{T h}\left(t_{2}, \operatorname{neg}\left([\Phi]^{c}\right)\right)\right]
$$

But the formula (3.3) contradicts the formula (3.1). Therefore: $T h \Vdash \operatorname{Pr}_{T h}\left([\neg \Phi]^{c}\right)$.

\section{Lemma3.2.}

Assume that: (i)Con(Th)and (ii) $\quad T h \vdash$ $\operatorname{Pr}_{T h}\left([\neg \Phi]^{c}\right)$, where $\Phi$ is a closed formula. Then $T h \sharp$ $\operatorname{Pr}_{T h}\left([\Phi]^{c}\right)$.

\section{Theorem3.1.}

[7],[8]. (Generalized Löb's Theorem). Assume that:Con(Th). Then theory Th can be extended to a maximally consistent nice theory $T h^{\#}$ over Th.

Proof.Let ., $\Phi_{1}, \ldots, \Phi_{i}, \ldots$ be an enumeration of all wff's of the theory Th (this can be achieved if the set of propositional variables can be enumerated). Define a chain

$$
\wp=\left\{T h_{i} \mid i \in \mathbb{N}\right\}, T h_{1}=T h,
$$

of the consistent theories inductively as follows: assume that theory $T h_{i}$ is defined.

(i)

$$
\text { Suppose that a statement (3.4) is satisfied }
$$

$$
\begin{gathered}
T h \vdash \operatorname{Pr}_{T h}\left(\left[\Phi_{i}\right]^{c}\right) \text { and } \\
{\left[T h \nvdash \Phi_{i}\right] \wedge\left[M M_{\omega}^{T h} \vDash \Phi_{i}\right]}
\end{gathered}
$$

Then we define theory $T h_{i+1}$ as follows:

$$
T h_{i+1} \triangleq T h_{i} \cup\left\{\Phi_{i}\right\}
$$

Suppose that a statement (3.5) is satisfied

$$
\begin{gathered}
T h \vdash \operatorname{Pr}_{T h}\left(\left[\neg \Phi_{i}\right]^{c}\right) \text { and } \\
{\left[T h \Vdash \neg \Phi_{i}\right] \wedge\left[M M_{\omega}^{T h} \vDash \neg \Phi_{i}\right] .}
\end{gathered}
$$

Then we define theory $T h_{i+1}$ as follows

$$
T h_{i+1} \triangleq T h_{i} \cup\left\{\neg \Phi_{i}\right\} .
$$

Suppose that a statement (3.6) is satisfied

$$
\begin{gathered}
T h \vdash \operatorname{Pr}_{T h}\left(\left[\Phi_{i}\right]^{c}\right) \text { and } \\
T h_{i} \vdash \Phi_{i} .
\end{gathered}
$$

Then we define theory $T h_{i+1}$ as follows:

$$
T h_{i+1} \triangleq T h_{i} \cup\left\{\Phi_{i}\right\}
$$

(iv) Suppose that a statement (3.7) is satisfied

$$
\begin{gathered}
T h \vdash \operatorname{Pr}_{T h}\left(\left[\neg \Phi_{i}\right]^{c}\right) \text { and } \\
T h_{i} \vdash \neg \Phi_{i} .
\end{gathered}
$$

Then we define theory $T h_{i+1}$ as follows: $T h_{i+1} \triangleq T h_{i}$. We define now theory $T h^{\#}$ as follows

$$
T h^{\#}=\bigcup_{i \in \mathbb{N}} T h_{i} .
$$

First, notice that each $T h_{i}$ is consistent. This is done by induction on $\mathrm{i}$ and by Lemmas 3.1-3.2. By assumption, the case is true when $i=1$. Now, suppose $T h_{i}$ is consistent. Then its deductive closure $\operatorname{Ded}\left(T h_{i}\right)$ is also consistent. If a statement (3.6) is satisfied, i.e. $T h \vdash \operatorname{Pr}_{T h}\left(\left[\Phi_{i}\right]^{c}\right)$ and $T h_{i} \vdash$ $\Phi_{i}$ then clearly $T h_{i+1} \triangleq T h_{i} \cup\left\{\Phi_{i}\right\}$ is consistent since it is a subset of closure $\operatorname{Ded}\left(T h_{i}\right)$.If a statement (3.7) is satisfied, i.e., $\quad T h \vdash \operatorname{Pr}_{T h}\left(\left[\neg \Phi_{i}\right]^{c}\right)$ and $T h_{i} \vdash \neg \Phi_{i}$ then clearly $T h_{i+1} \triangleq T h_{i} \cup\left\{\neg \Phi_{i}\right\}$ is consistent since it is a subset of closure $\operatorname{Ded}\left(T h_{i}\right)$.Otherwise:

(i) if a statement (3.4) is satisfied, i.e. $T h \vdash$ $\operatorname{Pr}_{T h}\left(\left[\Phi_{i}\right]^{c}\right)$ and $T h \nvdash \Phi_{i}$ then clearly $T h_{i+1} \triangleq$ $T h_{i} \cup\left\{\Phi_{i}\right\}$ is consistent by Lemma 3.1 and by one of the standard properties of consistency: $\Delta \cup\{A\}$ is consistent iff $\Delta \nvdash \neg A$;

(ii) if a statement (3.5) is satisfied, i.e. $T h \vdash$ $\operatorname{Pr}_{T h}\left(\left[\neg \Phi_{i}\right]^{c}\right)$ and $T h \Vdash \neg \Phi_{i}$, then clearly $T h_{i+1} \triangleq$ $T h_{i} \cup\left\{\neg \Phi_{i}\right\}$ is consistent by Lemma 3.2 and by one of the standard properties of consistency: $\Delta \cup\{\neg A\}$ is consistentiff $\Delta \nvdash \neg A$.

Next, notice $\operatorname{Ded}\left(T h^{\#}\right)$ is a maximally consistent nice extension of the set $\operatorname{Ded}(T h)$. A set $D e d\left(T h^{\#}\right)$ is consistent because, by the standard Lemma 3.3 below, it is the union of 
a chain of consistent sets. To see that $\operatorname{Ded}\left(T h^{\#}\right)$ is maximal, pick any wff $\Phi$. Then $\Phi$ is some $\Phi_{i}$ in the enumerated list of all wff's. Therefore for any $\Phi$ such that $T h \vdash \operatorname{Pr}_{T h}\left(\left[\Phi_{i}\right]^{c}\right)$ or $T h \vdash \operatorname{Pr}_{T h}\left(\left[\neg \Phi_{i}\right]^{c}\right)$ either $\Phi \in T h^{\#}$ or $\neg \Phi \in T h^{\#}$.

Since $\operatorname{Ded}\left(T h_{i+1}\right) \subseteq \operatorname{Ded}\left(T h^{\#}\right) \quad$ we have $\Phi \in$ $\operatorname{Ded}\left(T h^{\#}\right)$ or $\neg \Phi \in \operatorname{Ded}\left(T h^{\#}\right)$, which implies that $\operatorname{Ded}\left(T h^{\#}\right)$, is maximally consistent nice extension of the $\operatorname{Ded}(T h)$.

\section{Lemma3.3.}

The union of a chain $\wp=\left\{\Gamma_{i} \mid i \in \mathbb{N}\right\}$ of theconsistentsets $\Gamma$, ordered by $\subseteq$, is consistent.

\section{Definition3.4.}

(a) Assume that a theoryThhas $\omega$-model $M_{\omega}^{T h}$ and $\Phi$ is aTh-sentence. Let $\Phi_{\omega}$ be a Th-sentence $\Phi_{\text {with }}$ all quantifiers relativized to $\omega$-model $M_{\omega}^{T h}$ [9];

(b) Assume that a theory Thhas standard model $S M^{T h}$

And $\Phi$ is a Th-sentence. Let $\Phi_{S M}$ be a Th-sentence $\Phi$ with all quantifiers relativized to a model $S M^{T h}$ [9].

\section{Remark3.1.}

In some special cases we denote a sentence $\Phi_{\omega}$ by a symbol: $\Phi\left[M_{\omega}^{T h}\right]$.

\section{Definition3.5.}

(a)Assume that Thhas an $\omega$-model $M \stackrel{T h}{\omega}$. Let $T h_{\omega}$ be a theory Threlativized toa model $M_{\omega}^{T h}$ - i.e., any $T h_{\omega}$-sentence $\Psi$ has a form $\Phi_{\omega}$ for some Th-sentence $\Phi[9]$;

(b) Assume that Th has an standard model $S M^{T h}$. Let $T h_{S M}$ be a theory Th relativized to a model $S M^{T h}$ - i.e., any $T h_{S M}$-sentence $\Psi$ has a form $\Phi_{S M}$ for some Th-sentence $\Phi$ [9].

\section{Remark3.2.}

In some special cases we denote a theory $T h_{\omega}$ by a symbol:Th[$\left[M_{\omega}^{T h}\right]$.

Definition3.6.

(a) For a given $\omega$-model $M_{\omega}^{\mathrm{T}}$ of the Thand for any $T h_{\omega}$-sentence $\Phi_{\omega}$, we define relation $M{ }_{\omega}^{T h} \vDash * \Phi_{\omega}$ such that the nextequivalence:

$$
\begin{gathered}
M_{\omega}^{T h} \vDash * \Phi_{\omega} \text { iff }\left[T h^{\dagger} \vdash \Phi_{\omega}\right] \wedge \\
\wedge\left[\left(T h_{\omega} \vdash \operatorname{Pr}_{T h_{\omega}}\left(\left[\Phi_{\omega}\right]^{c}\right) \leftrightarrow T h^{\dagger} \vdash \Phi_{\omega}\right)\right],
\end{gathered}
$$

where $T h^{\dagger} \triangleq T h+\exists M_{\omega}^{T h}$ is satisfied.

(b) For a given standard model $S M^{T h}$ of the theoryThand for any $T h_{S M}$-sentence $\Phi_{S M}$ wedefine relation $M_{S M}^{T h} \vDash * \Phi_{S M}$ such that the next equivalence:

$$
\begin{gathered}
M_{S M}^{T h} \vDash * \Phi_{S M} \text { iff }\left(T h^{\dagger} \vdash \Phi_{S M}\right) \wedge \\
\wedge\left(T h_{\mathrm{SM}} \vdash \operatorname{Pr}_{T h_{S M}}\left(\left[\Phi_{S M}\right]^{c}\right) \leftrightarrow T h^{\dagger} \vdash \Phi_{S M}\right),
\end{gathered}
$$

where $T h^{\dagger} \triangleq T h+\exists M_{S M}^{T h}$, issatisfied.

Theorem3.2.(Strong Reflection Principle correspondingto $\omega$-model).Assume that: (i) Con(Th),(ii) Th has $\omega$-model $M{ }_{\omega}^{T h}$, i.e. $M{ }_{\omega}^{T h} \vDash T h_{\omega}$.Let $\Phi$ be a Th-sentence. Then

(a) $T h_{\omega} \vdash \operatorname{Pr}_{T h_{\omega}}\left(\left[\Phi_{\omega}\right]^{c}\right) \leftrightarrow T h_{\omega} \vdash \Phi_{\omega}$,

(b) $T h_{\omega} \vDash \operatorname{Pr}_{T h_{\omega}}\left(\left[\Phi_{\omega}\right]^{c}\right) \leftrightarrow T h_{\omega} \vDash \Phi_{\omega}(3.10)$

Proof.(a) Let $\Phi$ is any axiom of thetheory $T h$. Then statement (3.10) immediately follows from Definition 3.6 (a).The one direction is obvious. For the other, assume that

$T h_{\omega} \vdash \operatorname{Pr}_{T h_{\omega}}\left(\left[\Phi_{\omega}\right]^{c}\right),(3.11)$

$T h_{\omega} \Vdash \Phi_{\omega}$ and $T h_{\omega} \vdash \neg \Phi_{\omega}$. Then

$T h_{\omega} \vdash \operatorname{Pr}_{T h_{\omega}}\left(\left[\neg \Phi_{\omega}\right]^{c}\right) .(3.12)$

Notethat (i)+(ii) implies $\operatorname{Con}\left(T h_{\omega}\right)$. Let $\operatorname{Con}_{T h_{\omega}}$ be the formula:

$\operatorname{Con}_{T h_{\omega}} \triangleq \forall t_{1} \forall t_{2} \forall t_{3}\left(t_{3}=\left[\Phi_{\omega}\right]^{c}\right) \neg\left[\operatorname{Prov}_{T h_{\omega}}\left(t_{1},\left[\Phi_{\omega}\right]^{c}\right) \wedge\right.$ $\left.\operatorname{Prov}_{T h_{\omega}}\left(t_{2}, \operatorname{neg}\left(\left[\Phi_{\omega}\right]^{c}\right)\right)\right] \leftrightarrow(3.13)$

$\neg \exists t_{1} \neg \exists t_{2} \neg \exists t_{3}\left(t_{3}=\left[\Phi_{\omega}\right]^{c}\right)\left[\operatorname{Prov}_{T h_{\omega}}\left(t_{1},\left[\Phi_{\omega}\right]^{c}\right) \wedge\right.$ $\left.\operatorname{Prov}_{T h_{\omega}}\left(t_{2}, \operatorname{neg}\left(\left[\Phi_{\omega}\right]^{c}\right)\right)\right]$,

Here $t_{1}, t_{2}, t_{3}$ is a closed term. Note that in any $\omega$-model $M{ }_{\omega}^{\mathrm{T}}{ }^{\mathrm{b}}$ by the canonical observation one obtain the equivalence: $\operatorname{Con}\left(T h_{\omega}\right) \leftrightarrow \operatorname{Con}_{T h_{\omega}}$, But the formulae: (3.11) (3.12)contradicts the formula (3.13). Therefore $T h_{\omega} \Vdash \Phi_{\omega}$ and $T h_{\omega} \nvdash \operatorname{Pr}_{T h_{\omega}}\left(\left[\neg \Phi_{\omega}\right]^{c}\right)$.

Then a theory $T h_{\omega}^{\prime}=T h_{\omega}+\neg \Phi_{\omega}$ is consistent and from the above observation one have obtain that:

$$
\begin{gathered}
\operatorname{Con}\left(T h_{\omega}^{\prime}\right) \leftrightarrow \operatorname{Con}_{T h_{\omega}^{\prime}}, \text { where } \\
\operatorname{Con}_{T h_{\omega}^{\prime} \leftrightarrow} \leftrightarrow \\
\leftrightarrow \neg \exists t_{1} \neg \exists t_{2} \neg \exists t_{3}\left(t_{3}=\left[\Phi_{\omega}\right]^{c}\right)\left[\operatorname{Prov}_{T h_{\omega}^{\prime}}\left(t_{1},\left[\Phi_{\omega}\right]^{c}\right) \wedge\right. \\
\left.\operatorname{Prov}_{T h_{\omega}^{\prime}}\left(t_{2}, \operatorname{neg}\left(\left[\Phi_{\omega}\right]^{c}\right)\right)\right],
\end{gathered}
$$

On the other hand one obtain

$$
T h_{\omega}^{\prime} \vdash \operatorname{Pr}_{T h_{\omega}^{\prime}}\left(\left[\Phi_{\omega}\right]^{c}\right), T h_{\omega}^{\prime} \vdash \operatorname{Pr}_{T h_{\omega}^{\prime}}\left(\left[\neg \Phi_{\omega}\right]^{c}\right)
$$

But the formulae (3.15), contradicts the formula (3.14). This contradiction completed the proof.

(b) The one direction is obvious. For the other, assume that:(i)Th $h_{\omega} \vDash \operatorname{Pr}_{T h_{\omega}}\left(\left[\Phi_{\omega}\right]^{c}\right)$ and $(i i) T h_{\omega} \vDash \neg \Phi_{\omega}$. From (ii) using derivability condition D1 (see Remark 2.1) one obtain $T h_{\omega} \vDash \operatorname{Pr}_{T h_{\omega}}\left(\left[\neg \Phi_{\omega}\right]^{c}\right)$. Therefore one obtainthe contradiction

$$
T h_{\omega} \vDash \operatorname{Pr}_{T h_{\omega}}\left(\left[\Phi_{\omega}\right]^{c}\right) \wedge \operatorname{Pr}_{T h_{\omega}}\left(\left[\neg \Phi_{\omega}\right]^{c}\right) .
$$

This contradiction completed the proof.

Definition3.7.(a)Assume that: (i) Thhas an $\omega$-model $M_{\omega}^{T h}$,(ii) $M_{\omega, \vDash}^{T h}$ is a set and (iii) $M_{\omega}^{T h} \vDash * T h_{\omega}$. Then we 
said that $M{ }_{\omega}^{T h}$ is a strong $\omega$-model of the $T h$ and denote such $\omega$-model of the $T h \operatorname{as} M_{\omega, \text { } * \text { * }}^{T h}$.

(b) Assume that: (i) Thhas an standard modelSM ${ }^{T h}$,(ii) $S M_{\models *}^{T h}$ is a set and (iii)SM$M^{T h} \vDash * T h_{S M}$. Then we said that $S M^{T h}$ is a strong standard model of the $T h$ and denote such standard model of the $T h$ as $S M_{\models *}^{T h}$.

Remark3.3. Note that there exists formal theoriesThwhich has not a strong standard models.For example a theory $Z F C+(V=L)$ has not any strong standard model.

Definition3.8.(a) Assume that Thhas a strong $\omega$-model $M_{\omega, \vDash *}^{T h}$. Then we said that This a strongly consistent.

(b) Assume that $T h$ has a strong standard model $S M_{\text {F* }}^{T h}$

Then we said that Th is a strongly SM-consistent

Definition3.9.(a) Assume that a theoryThhas a strong $\omega$-model $M_{\omega \text {, } \text {, }^{T}}^{T h}$. and $\Phi$ is a $T h$-sentence. Let $\Phi_{\omega, \text {, * }}$ be a $T h$-sentence $\Phi$ with all quantifiers relativized to a strong $\omega$-model $M_{\omega, \text {, } * \text { * }}^{T h}$

(b) Assume that $T h$ has a strong standard model $S M_{\vDash *}^{T h}$ and $\Phi$ is a $T h$-sentence Let $\Phi_{S M, \vDash *}$ be a $T h$-sentence $\Phi$ with all quantifiers relativized toa model $S M_{\vDash *}^{T h}$.

\section{Remark3.4.}

In some special cases we denote a sentence $\Phi_{\omega, \text { 上* }}$ by a symbol: $\Phi\left[M_{\omega, \vDash *}^{T h}\right]$.

Definition3.10. Assume that a theoryThhas a strong $\omega$-model $M_{\omega, \vDash * *}^{T h}$. Let $T h_{\omega, \vDash *}$ be a theory Threlativised to a model $M_{\omega, \vDash *}^{T h}$ i.e., any $T h_{\omega, \vDash *}$-sentence $\Psi$ has a form $\Phi_{\omega, \text {, * }}$ for some $T h$-sentence $\Phi$.

\section{Remark3.5.}

In some special cases we denote a theory $T h_{\omega, \vDash *}$ by a symbol:Th $\left[M_{\omega, \vDash *}^{T h}\right]$.

\section{Definition3.11.}

(a) Let Thbe a theory such that Assumption 2.1 is satisfied. Let $\widehat{\operatorname{Con}}\left(T h ; M_{\omega, \vDash *}^{T h}\right)$ be a predicate in Thasserting that $M_{\omega, \vDash *}^{T h}$ is a strong $\omega$-model of the Th.Then a tenceCon $\left(T h ; M_{\omega, \text { } Е *}^{T h}\right)$ such that

$$
\operatorname{Con}\left(T h ; M_{\omega, \vDash *}^{T h}\right) \leftrightarrow \exists M_{\omega, \vDash *}^{T h} \widehat{\operatorname{Con}}\left(T h ; M_{\omega, \vDash *}^{T h}\right)
$$

isa sentence in $T h$ asserting that $T h$ has a strong

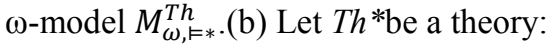

$$
T h^{*}=T h+\operatorname{Con}\left(T h ; M_{\omega, \text { Е* }}^{T h}\right)
$$

$\operatorname{Let} \operatorname{Con}\left(T h^{*} ; M_{\omega, \vDash *}^{T h^{*}}\right)$ be a sentence in $T h^{*}$ asserting that $T h^{*}$ has a strong $\omega$-model $M_{\omega, \text {, } * \text {. }}^{T h^{*}}$.

Lemma3.4.Assumethat a theory $T h$ has a strong $\omega$-model $S M_{\omega, \text {, } *}^{T h}$ and a theory $T h^{*}$ has a strong $\omega$-model $M_{\omega, \models *}^{T h^{*}}$.Then: $(i)$ a sentenceCon $\left(T h ; M_{\omega, \vDash *}^{T h}\right)$ is aTh-sentence, (ii);a sentence $\operatorname{Con}\left(T h^{*} ; M_{\omega \text {, }}^{T h^{*}}\right)$ is a $T h^{*}$-sentence.

Proof.Immediately follows from Definition 3.6 and Definition 3.11 .

Assumption3.1. We now assume, throughout this subsec- tion that $T h$ is a strongly consistent, i.e. a tence $\operatorname{Con}\left(T h ; M_{\omega, \vDash *}^{T h}\right)$ is true in any $\omega$-model $M_{\omega}^{T h}$ of the Th.

\section{Remark3.6.}

Note that:

$$
\operatorname{Con}\left(T h ; M_{\omega, \vDash *}^{T h}\right) \leftrightarrow \operatorname{Con}_{T h_{\omega, \vDash *}}
$$

where

$$
\operatorname{Con}_{T h_{\omega, \vDash *}} \leftrightarrow \neg \operatorname{Pr}_{T h_{\omega, \vDash *}}\left(\left[\Phi_{\omega, \vDash *}\right]^{c}\right)
$$

Herea sentence $\Phi_{\omega, \vDash *}$ is refutablein $T h_{\omega, \vDash *}$.

Remark3.6.Note that:

$$
\operatorname{Con}\left(T h^{*} ; M_{\omega, \vDash *}^{T h^{*}}\right) \leftrightarrow \operatorname{Con}_{T h_{\omega}^{*}}
$$

where

$$
\operatorname{Con}_{T h_{\omega, \models *}^{*}} \leftrightarrow \neg \operatorname{Pr}_{T h_{\omega, \models *}^{*}}\left(\left[\Phi_{\omega, \vDash *}^{*}\right]^{c}\right) .
$$

Herea sentence $\Phi_{\omega, \vDash *}^{*}$ is refutablein $T h_{\omega, \vDash *}^{*}$.

\section{Lemma3.5.}

Ander Assumption3.1 a theory Th* is a strongly consistent.

Proof. Assume that a theoryTh*is no strongly consistent, that is, has not any strong $\omega$-model $M_{\omega, \text {, * }}^{T h^{*}}$ of the Th*. This means that there is no any model $M^{T h}$ of the theory Th in which a sentence $\operatorname{Con}\left(T h ; M_{\omega, \text {, } *}^{T h}\right)$ is true and therefore a sentence $\neg \operatorname{Con}\left(T h ; M_{\omega, \text {, } *}^{T h}\right)$ is true in any model $M^{T h}$ of the theoryTh.In particular a sentence $\Theta$ :

$\Theta \triangleq \neg \operatorname{Con}\left(T h ; M_{\omega, \vDash *}^{T h}\right)$

(3.20)is true in any strong $\omega$-model $M_{\omega, \text { 上* }}^{T h}$ of theTh.Therefore from formula (3.16) one obtain, that a formula $\neg \operatorname{Con}_{T h_{\omega, \text { Е* }}}$ is true in any strong $\omega$-model $M_{\omega \text {,Е* }}^{T h}$ oftheTh,i.e.

$$
\widetilde{M}_{\omega, \vDash *}^{T h} \vDash \neg \operatorname{Con}_{\widetilde{T h}_{\omega, \vDash *}}
$$

Here $\widetilde{T h}_{\omega, \vDash *} \triangleq T h_{\omega, \vDash *}\left[\widetilde{M}_{\omega, \text { Е* }}^{T h}\right]$, i.e. $\widetilde{T h}_{\omega, \vDash *}$ is a theory $T h_{\omega, \vDash *}$ relativized to a strong $\omega$-model $\widetilde{M}_{\omega, \vDash *}^{T h}$, seeRemark3.2.From formulae (3.17) and (3.21) one obtain

$$
\widetilde{M}_{\models *}^{T h} \vDash \operatorname{Pr}_{\widetilde{T h}_{\omega, \vDash *}}\left(\left[\widetilde{\Phi}_{\omega, \vDash *}\right]^{c}\right) .
$$

Here $\quad \widetilde{\Phi}_{\omega, \vDash *} \triangleq \Phi_{\omega, \vDash *}\left[\widetilde{M}_{\omega, \vDash *}^{T h}\right]$, i.e. $\widetilde{\Phi}_{\omega, \vDash *}$ is a sentence $\Phi_{\omega, \vDash *}$ relativized to a strong $\omega$-model $\widetilde{M}_{\omega, \models *}^{T h}$, see Remark 3.1.So from formula (3.22) using a Strong Reflection Principle (Theorem3.2.b) one obtain

$\widetilde{M}_{\omega, \vDash *}^{T h} \vDash \widetilde{\Phi}_{\omega, \vDash *}$, where a sentence $\widetilde{\Phi}_{\omega, \vDash * *}$ is refutable in a theory $\widetilde{T h}_{\omega, \vDash *}$, i.e. $\widetilde{T h}_{\omega, \vDash *} \vdash \neg \widetilde{\Phi}_{\omega, \vDash *}$ andtherefore $\widetilde{M}_{\omega, \vDash *}^{T h} \vDash$ $\neg \widetilde{\Phi}_{\omega, \vDash *}$.

Thus a sentence $\widetilde{\Theta}_{1} \triangleq \widetilde{\Phi}_{\omega, \vDash *} \wedge \neg \widetilde{\Phi}_{\omega, \vDash *}$ is satisfiedina model $\widetilde{M}_{\omega, \vDash *}^{T h}$, i.e. $\widetilde{M}_{\omega, \vDash *}^{T h} \vDash \widetilde{\Theta}_{1}$. But a sentence $\widetilde{\Theta}_{1}$ contrary to the assumption that $T h$ is a strongly consistent. This contradiction completed the proof. 


\section{Theorem3.3.}

$T h$ has notany strong $\omega$-model $M_{\omega, \models *}^{T h}$.Proof. By Lemma 3.5 and formula (3.17) one obtain that $T h_{\omega, \models *}^{*} \vdash \operatorname{Con}_{T h_{\omega, \models *}^{*}}$. But Gödel's Second Incompleteness Theorem applied to $T h_{\omega, \models *}^{*}$ asserts that a sentence $\operatorname{Con}_{T h_{\omega, \text { E* }}^{*}}$ is in $T h_{\omega, \vDash *}^{*}$. This contradiction completed the proof.

\section{Theorem3.4.}

$Z F C$ has not any strong $\omega$-model $M_{\omega, \vDash *}^{Z F C}$.

Proof. Immediately follows from Theorem 3.3 and definitions.

\section{Theorem3.5.}

$Z F C$ has not any strong standard model.SMF*

Proof. Immediately follows from Theorem 3.4 and definitions.

\section{Theorem3.6.}

$Z F C+\operatorname{Con}(Z F C)$ is incompatible with all the usual large cardinal axioms [10],[11] which imply the existence of a strong standard model of $Z F C$.

Proof.Theorem3.6 immediately follows from Theorem 3.5 .

\section{Lemma3.6.}

Let $\kappa$ be an inaccessible cardinal and let $H_{\kappa}$ be a set of all sets having hereditary size less then, $\kappa$.Suppose that $\operatorname{Con}(Z F C+\exists \kappa)$. Then $H_{\kappa}$ forms a strong standard model of $Z F C$.

Proof. From definitions one obtain that $H_{\kappa}$ forms some standard model $S M$ of $Z F C$. Let $\varphi^{Z F C}$ be any axiom of ZFC and $T h^{\dagger} \triangleq T h+\exists H_{\kappa}$. Thenbydefinitions one obtain

$$
\begin{gathered}
\left(T h^{\dagger} \vdash \varphi^{Z F C}\left[H_{\kappa}\right]\right) \wedge \\
\wedge\left(T h_{S M} \vdash \varphi^{Z F C}\left[H_{\kappa}\right] \leftrightarrow T h^{\dagger} \vdash \varphi^{Z F C}\left[H_{\kappa}\right]\right) .
\end{gathered}
$$

Using Strong Reflection Principle (see Theorem3.2) from statement (3.23)one obtain that RHS of the formula (3.9.b) is satisfied. Thus $H_{\kappa} \vDash * Z F C$.

\section{Theorem3.7.}

Let火 be an inaccessible cardinal. Then $\neg \operatorname{Con}(Z F C+\exists \kappa)$.

Proof. Let $H_{\kappa}$ be a set of all sets having hereditary size less then $\kappa$.From Lemma3.6 we know that $H_{\kappa}$ forms a strong standard model of $Z F C$. Therefore Theorem 3.7 immediately follows from Theorem 3.6.

New Foundation [NF for short] was introduced by Quine [13].It well known that his approach for blocking paradoxes of naïve set theory was to introduced a special stratification condition in the comprehension schema. Jensen [14] introduced $N F U$, the [slight?]version of $N F$ in which axiom of the extensionality was weakened to allow ur-elements which are not sets. The theory $N F U$ has a universal class, $V$, which contains all of its subsets.

\section{Definition3.12.}

We say that a set $S$ is a Cantorianiffthere is a bijection of $S$ with the set $U S C(S)$ consistingof all the singletons whose members lie in $S$. A set is strongly Cantorianiff the map $x \mapsto\{x\}$ provides a bijection of $S$ withaset $U S C(S)$.

Holmes [15],[16],[17]introduced the system NFUA which is obtained from $N F U$ by adjoining the axiom that:"EveryCantorian set is a strongly Cantorian."

Theorem3.8.(Solovay,1995)[18].The following theories are equiconsistent:

(i) $T h_{1} \triangleq \mathrm{ZFC}+\{$ "there is n-Mahlo cardinal": $n \in \omega\}$,

(ii) $T h_{2} \triangleq N F U A$.

Holmes also introduced stronger theory NFUB [19].Note that in $N F U$ one can introduce ordinal such that any ordinal $\mathcal{R}$ consists of the class of all well-orderings which are order-isomorphic to a given well-ordering.

\section{Definition3.13.}

We say that an ordinal $\mathfrak{S}$ is Cantorian if the underlying sets of the well-orderings which are its members are all Cantorian.

\section{Definition3.14.}

We say thata subcollection $\Sigma$ of the Cantorian ordinals is coded if there is there is some set $\sigma$ whose members among the Cantorian ordinals are precisely the members of $\Sigma$.

The system NFUB is obtained from the system NFUA by adding the axiom schema which asserts that any subcollection $\Sigma$ of the Cantorian ordinals which is definable by a formula of the language of $N F U B$ [possible unstratified and possible with parameters] is coded by some set $\sigma$.

\section{Theorem3.9.}

(Solovay,1997)[19].Let $Z F C^{-}$be a theory consisting all the axioms of ZFC except the power set axiom. The following theories are equiconsistent:

(i) $T h_{1} \triangleq$ $Z F C^{-}+\{$there is a weakly compact cardinal $\}$,

(ii) $T h_{2} \triangleq N F U B$.

\section{Remark3.7.}

The formulation of "weak compactness"we shall use is: $\kappa$ is weakly compact if $\kappa$ is strongly inaccessible and every $\kappa$-tree has a branch [19].

\section{Theorem3.10. $\neg$ Con (NFUA).}

Proof. Theorem3.10 immediately follows from Theorem3.7 and Theorem3.8.

\section{Theorem3.11. $\neg$ Con $(N F U B)$}

Proof.Theorem3.11 immediately follows from Theorem3.7, Theorem3.9 and definitions.

Strong Reflection Principle Corresponding to Nonstandard Models. LetTh be consistent formal theory. When in- 
terpretedas a

proof within first order theory $T h$,Dedekind's categoricity proof for $P A$ shows that the each model $M^{T h}$ of the $T h$ has the unique sub-model $M^{P A} \subset M^{Z F C} \subseteq M^{T h}$ of the $P A$ arithmetic, up to isomorphism, that imbedsas an initial segment of all models of $P A$ contained within model $M^{Z F C}$ of set theory $Z F C$.In the standard model of the $T h$ this smallest model of the $P A$ is the standard model $S M^{P A} \approx \mathbb{N}$ of $P A$.

\section{Remark4.1.}

Note that.in any nonstandard model $N S M^{T h}$ of the Thit may by a nonstandard model $N S M^{P A}$ of the $P A$.

\section{Remark4.2.}

Note thatin any nonstandard model of the $P A$, the terms $\overline{0}, S \overline{0}=\overline{1}, S S \overline{0}=\overline{2}, \ldots$ comprise the initial segment isomorphic to $S M^{P A}$. This initial segment is called the standard cutThe order type of any nonstandard model of $P A$ is equal to $\mathbb{N}+\mathrm{A} \times \mathbb{Z}$ for some linear order $\mathrm{A}[12]$.

\section{Definition4.1.}

Let $N S M^{T h}$ bea nonstandard model of the $T h$ and $\Phi$ is a $T h$-sentence. Let $\Phi_{N S M}$ be a Th-sentence $\Phi$ with all quantifiers relativized to nonstandardmodel $N s M^{T h}$.In somespecial cases we denote thissentence by symbol $\Phi\left[N S M^{T h}\right]$.

Definition4.2.Let $T h_{N S M}$ be a theory $T h$ relativized to a model $N s M^{T h}$.In some special cases we denote this theory as:Th[NsM $\left.M^{T h}\right]$

\section{Definition4.3.}

One can define a predicate $\operatorname{Pr}_{T h_{N S M}}(y)$ such that for all $\mathrm{y} \in N s M^{T h}$ theequivalence::

$$
\operatorname{Pr}_{T h_{N S M}}(y) \leftrightarrow \exists x\left(x \in M^{P A}\right) \operatorname{Prov}_{T h_{N S M}}(x, y) .
$$

is satisfied.Therefore one obtain a predicate asserting provability in a theory $T h_{N S M}$.

\section{Definition4.4.}

For a given nonstandard model $N s M^{T h}$ of the Thand any $T h_{N S M}$-sentence $\Phi_{N S M}$ we define relation $N s M^{T h} \vDash *$ $\Phi_{N S M}$ such the next equivalence:

$$
\begin{gathered}
N s M^{T h} \vDash * \Phi_{N S M} \text { iff }\left(T h^{\dagger} \vdash \Phi_{N S M}\right) \wedge \\
\wedge\left[\left(T h_{S M} \vdash \operatorname{Pr}_{T h_{N S M}}\left(\left[\Phi_{N S M}\right]^{c}\right)\right) \leftrightarrow\left(T h^{\dagger} \vdash \Phi_{N S M}\right)\right],
\end{gathered}
$$

where $T h^{\dagger} \triangleq T h+\exists N s M^{T h}$, issatisfied.

Theorem4.1.(StrongReflectionPrinciple corresponding to nonstandard model)Assume that:(i) Con(Th),(ii)Th has annonstandard model $N s M^{T h}$, i.e. $N s M^{T h} \vDash T h_{N s M}$. Let $\Phi$ is a Th-sentence. Then

$$
\operatorname{Pr}_{T h_{N S M}}\left(\left[\Phi_{N S M}\right]^{c}\right) \leftrightarrow T h_{N S M} \vdash \Phi_{N S M} .
$$

Proof. The proof completely to similarly a proof of the Theorem 3.2.
Definition4.5.Assume that: (i) Th has an nonstandard model $N s M^{T h}$, (ii) $N s M_{\text {F* }}^{T h}$ is a set and $(i i) N s M^{T h} \vDash *$ $\Phi_{N s M}$. Then we said that $N s M^{T h}$ is astrong nonstandard model of the $T h$ anddenotesuchnonstandard model as $N s M_{\vDash *}^{T h}$.

\section{Definition4.6.}

Assume that a theoryTh has astrononstandard el $N s M_{F *}^{T h}$. Then we said that a theory $\mathrm{Th}$ is a strongly NsM-consistent.

\section{Definition4.7.}

Assume that a theory $T h$ has a strongnonstandard el $N s M_{\vDash *}^{T h}$ and $\Phi$ is a $T h$-sentence. Let $\Phi_{N s M, \vDash *}$ be a $T h$-sentence $\Phi$ with all quantifiers and all constants relativized to a model $N s M_{\models *}^{T h}$.

\section{Remark4.3.}

In some special cases we denote a sentence $\Phi_{N s M, \vDash *}$ by a symbol: $\Phi\left[N s M_{\vDash *}^{T h}\right]$.

\section{Definition4.8.}

Assume that a theory Th has a strong nonstandard el $N S M_{\models *}^{T h}$. Let $T h_{N S M, \vDash *}$ be a theory $T h$ relativized to a model $N s M_{\vDash *}^{T h}$ i.e., any $T h_{N S M, \vDash *}$-sentence $\Psi$ has a form $\Phi_{N S M, \vDash *}$ for some Th-sentence $\Phi$.

Remark4.4.In some special cases we denote

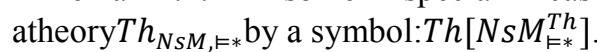

\section{Assumption4.1.}

We now assume throughout this subsectionthat $T h$ is a strongly $N s M$-consistent, i.e. a sentenceCon $\left(T h ; N s M_{\models *}^{T h}\right)$ is true in any nonstandard model $N s M^{T h}$ of the $T h$.

Definition4.9.(a) Let $T h$ be a theory such that Assumption 3.1 is satisfied. Let $\widehat{\operatorname{Con}}\left(T h ; N s M_{\models *}^{T h}\right)$ be a predicate in Thasserting that $N S M_{\vDash *}^{T h}$ is a strong nonstandard model of the $T h$. Then a sentence $\operatorname{Con}\left(T h ; N s M_{\models *}^{T h}\right)$ such that:

$$
\operatorname{Con}\left(T h ; N s M_{\vDash *}^{T h}\right) \leftrightarrow \exists N s M_{\vDash *}^{T h} \widehat{\operatorname{Con}}\left(T h ; N s M_{\models *}^{T h}\right)(4.4)
$$

is a sentence in Thasserting that $T h$ has a strong non-standard model $N s M_{\models *}^{T h}$. (b) Let $T h^{*}$ be a theory $T h^{*}=T h+\operatorname{Con}\left(T h ; N s M_{F *}^{T h}\right)$.Let $\operatorname{Con}\left(T h^{*} ; N s M_{F *}^{T h^{*}}\right)$ bea sentence in $T h^{*}$ asserting that $T h^{*}$ has a strongnonstandard $\operatorname{model} N s M_{\models *}^{T h}$.

\section{Lemma4.1.}

Assume that a theory $T h$ has a strong nonstandard model $N s M_{\models *}^{T h}$ anda theory $T h^{*}$ has a strong nonstandard el $N s M_{\models *}^{T h^{*}}$. Then: (i) a sentence $\operatorname{Con}\left(T h ; N S M_{\models *}^{T h}\right)$ is aTh-sentence, (ii)a sentence $\operatorname{Con}\left(T h^{*} ; N s M_{\text {上* }}^{T h^{*}}\right)$ is a $T h{ }^{*}$-sentence.

Proof.Immediately follows from Definition 4.4 and Definition 4.9.

Remark4.5.Note that: 


$$
\operatorname{Con}\left(T h ; N s M_{\vDash *}^{T h}\right) \leftrightarrow \operatorname{Con}_{T h_{N S M, \vDash *}},
$$

where

$$
\operatorname{Con}_{T h_{N S M, \vDash *}} \leftrightarrow \neg \operatorname{Pr}_{T h_{N S M, \vDash *}}\left(\left[\Phi_{N S M, \vDash *}\right]^{c}\right) .
$$

Here a sentence $\Phi_{N S M, \vDash * *}$ is refutable in $T h_{N S M, \vDash *}$.

\section{Remark4.6.}

Note that

$$
\operatorname{Con}\left(T h^{*} ; N s M_{\models *}^{T h^{*}}\right) \leftrightarrow \operatorname{Con}_{T h^{*}}{ }_{N S M, \vDash *^{\prime}}
$$

where

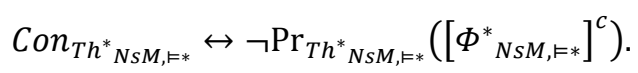

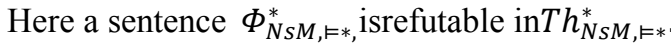

Lemma4.2.Ander Assumption 4.1 a theoryTh* is a strongly $N s M$-consistent.

Proof. The proof uses formulae (4.5) and (4.6) and completely, to similarly a proof of the Lemma 3.5 .

\section{Theorem4.2.}

Th has not any strong nonstandard model $N s M_{E^{*} *}^{T h}$.

Proof. The proof uses formulae (4.7) and (4.8) and completely, to similarly a proof of the Theorem 3.3.

Theorem4.3.ZFChas not any strong nonstandard elNSM $M_{E *}^{Z F C}$.

Proof. Immediately follows from theorem 4.2 and definitions.

Definability in Second-Order Set Theory

\section{Definition5.1.}

Assume that: (i) Con(Th) and (ii)Th has an $\omega$-model $M_{\omega}^{T h}$. Let $\Psi(X)$ be one-place open Th-wiff and let $\Psi_{\omega}(X)$ be $T h$-wiff $\Psi(x)$ relativized to $\omega$-model $M_{\omega}^{T h}$. Assume that condition

$$
T h_{\omega} \vdash \exists ! X_{\Psi}\left(X_{\Psi} \in M_{\omega}^{T h}\right)\left[\Psi_{\omega}\left(X_{\Psi}\right)\right]
$$

is satisfied. We say that an $T h$-wiff $\Psi(X)$ is a nice $T h$-wiff, iff condition (5.1) is satisfied.

\section{Definition5.2.}

Let us define a second-order predicate

$$
\begin{aligned}
& \Xi_{\omega}\left(\Psi\left(X_{\Psi}\right)\right) \text { suchthat equivalence } \\
& \Xi_{\omega}\left[\Psi\left(X_{\Psi}\right)\right] \leftrightarrow T h_{\omega} \vdash \Delta_{\omega}^{\Psi\left(X_{\Psi}\right)},
\end{aligned}
$$

where

$$
\Delta_{\omega}^{\Psi\left(X_{\Psi}\right)} \triangleq \exists ! X_{\Psi}\left(X_{\Psi} \in M_{\omega}^{T h}\right)\left[\Psi_{\omega}\left(X_{\Psi}\right)\right]
$$

is satisfied.We say that a set $Y$ is a $T h_{\omega}$-set iffthe second-order sentence:

$$
\Sigma^{Y}\left[\Psi(X), X_{\Psi}\right] \triangleq
$$

$$
\exists \Psi\left(X_{\Psi}\right)\left\{\Xi_{\omega}\left[\Psi\left(X_{\Psi}\right)\right] \wedge\left(Y=X_{\Psi}\right)\right\}
$$

is satisfied in $\omega$-model $M_{\omega}^{T h}$, i.e.

$$
M_{\omega}^{T h} \vDash \exists \Psi\left(X_{\Psi}\right)\left\{\Xi_{\omega}\left[\Psi\left(X_{\Psi}\right)\right] \wedge\left(Y=X_{\Psi}\right)\right\} .
$$

\section{Definition5.3.}

Let $\Psi_{1}(x)$ and $\Psi_{2}(x)$ is a nice Th-wiffs. Let us define equivalence relation $\Psi_{1}(x) \backsim \Psi_{2}(x)$ such that condition

$$
\Psi_{1}(x) \backsim \Psi_{2}(x) \leftrightarrow X_{\Psi_{1}}=X_{\Psi_{2}}
$$

is satisfied.

\section{Assumption5.1.}

Weassume now that: (i) a theory $T h$ admitscanonical primitive recursive encoding of syntax and (ii) the set of codes of axiom of This primitive recursive.

Lemma5.1.Second-order predicate $\Xi_{\omega}\left[\Psi\left(X_{\Psi}\right)\right]$ can be replaced by some equivalent first-order predicate:

$$
\check{\Xi}_{\omega}\left\{\left[\Psi\left(X_{\Psi}\right)\right]^{c},\left[X_{\Psi}\right]^{c}\right\} .
$$

Proof. Let us rewrite a sentence (5.3) in equivalent form such that

$$
\Delta_{\omega}^{\Psi\left(X_{1}\right)} \triangleq \exists ! X_{1}\left(X_{1} \in M_{\omega}^{T h}\right)\left[\Psi_{\omega}\left(X_{1}\right)\right] .
$$

Using a Strong Reflection Principle [formula (3.10.a)], one obtain equivalence

$$
\operatorname{Pr}_{T h_{\omega}}\left(\left[\Delta_{\omega}^{\Psi(X \Psi)}\right]^{c}\right) \leftrightarrow T h_{\omega} \vdash \Delta_{\omega}^{\Psi(X \Psi)} .
$$

Therefore

$$
\check{\Xi}_{\omega}\left\{\left[\Psi\left(X_{\Psi}\right)\right]^{c},\left[X_{\Psi}\right]^{c}\right\} \leftrightarrow \operatorname{Pr}_{T h_{\omega}}\left(\left[\Delta_{\omega}^{\Psi\left(X_{\Psi}\right)}\right]^{c}\right)
$$

Formula (5.9) andDefinition 5.2 completed the proof.Lemma5.2.Second-orderpredicate $\Sigma^{Y}\left[\Psi(X), X_{\Psi}\right]$ can be replaced by some equivalent first-order predicate:

$$
\breve{\Sigma}^{Y}\left[\left[\Psi\left(X_{\Psi}\right)\right]^{c},\left[X_{\Psi}\right]^{c},[Y]\right](5.10)
$$

Proof. Let us rewrite formula(5.4) in the next equivalent form

$$
\begin{gathered}
\Sigma^{Y}\left[\Psi\left(X_{1}\right)\right] \triangleq \\
\exists \Psi\left(X_{1}\right)\left\{\Xi_{\omega}\left[\Psi\left(X_{1}\right)\right] \wedge\left(Y=X_{1}\right)\right\}
\end{gathered}
$$

Using formula (5.7) one can rewrite RHS in the next equivalent first-order form

$$
\exists \mathrm{t}\left(\mathrm{t}=\left[\Psi\left(X_{1}\right)\right]^{c}\right)\left[\check{\Xi}_{\omega}\left\{\mathrm{t},\left[X_{1}\right]^{c}\right\} \wedge\left([Y]^{c}=\left[X_{1}\right]^{c}\right)\right] .
$$

Formula (5.12) completed the proof.

\section{Remark5.2.}

We now assume, throughout this subsection that encoding $[\circ]^{c}$ means canonical Gödel encoding such that defined in 
[20].Let $(i) \operatorname{EVbl}(\mathrm{x})$ be the predicate: $\mathrm{x}$ is a Gödel number of an expression consisting of a variable, $(i i) \operatorname{Fr}(\mathrm{y}, \mathrm{x})$ be the predicate:y is the Gödel number of 1-place openwffof $T h$ which contains free occurrences of the variable with Gödelnumber $\mathrm{x}[20]$.

\section{Remark5.3.}

Note that by using Remark 5.2, first-order predicate

$$
\check{\Xi}_{\omega}\left\{\left[\Psi\left(X_{1}\right)\right]^{c},\left[X_{1}\right]^{c}\right\},
$$

one can by replace in equivalent form such that

$$
\begin{gathered}
\breve{\Xi}_{\omega}\left\{y_{1}, x_{1}\right\}, \\
\text { where } M_{\omega}^{T h} \vDash \operatorname{Fr}\left(y_{1}, x_{1}\right) .
\end{gathered}
$$

\section{Remark5.4.}

Note that by using Remark 5.2 first-order predicate given by formula $(5.12)$ one can replace by first-order predicate such that

\section{Conclusion}

In this paper we proved so-called strong reflection principles corresponding to formal theories $T h$ which has $\omega$-models $M_{\omega}^{T h}$ and in particular to formal theories $T h$, which has a standard models $S M^{T h}$. Theassumption that there existsa standard model of $T h$ is stronger than the assumption that there exists a model of $T h$. This paper examined some specified classes of the standard and nonstandard models of $Z F C$ so-called strongstandardmodels of $Z F C$ and strong nonstandard models of ZFC correspondingly. Such strong standard models of $Z F C$ correspond tolarge cardinal axioms. In particular we proved that theo$\operatorname{ry} Z F C+\operatorname{Con}(Z F C)$ is incompatible with existence of any inaccessible cardinal $\kappa$.Note that the statement: $\operatorname{Con}(Z F C+\exists$ some inaccessible cardinal $\kappa)$ is $\Pi_{1}^{0}$.Thus Theorem 3.6 asserts there is exist numerical counterexample which is turn would imply that a specific polynomial equation has at least one integer root.

\section{References}

[1] M. H. Löb, "Solution of a Problem of Leon Henkin," The Journal of Symbolic Logic, Vol. 20, No. 2, 1955, pp. 115-118.

[2] C. Smorynski, "Handbook of Mathematical Logic," Edited by J. Barwise, North-Holland Publishing Company, 1977, p.1151.

[3] T. Drucker, "Perspectives on the History of Mathematical Logic,” Boston, Birkhauser, 2008, p.191.
[4] A. Marcja, C. Toffalori, "A Guide to Classical and Modern Model Theory," Springer, 2003. 371 p. Series: Trends in Logic, Vol. 19.

[5] F. W. Lawvere, C. Maurer, G. C. Wraith," Model Theory and Topoi." ISBN: 978-3-540-07164-8.

[6] D. Marker, "Model Theory: an Introduction," Graduate Texts in Mathematics, Vol. 217, Springer 2002.

[7] J.Foukzon, “GeneralizedLöb's Theorem. Strong Reflection Principles and Large Cardinal Axioms" http://arxiv.org/abs/1301.5340

[8] J. Foukzon, "An Possible Generalization of the Löb's Theorem," AMS Sectional Meeting AMS Special Session. Spring Western Sectional Meeting University of Colorado Boulder, Boulder, CO 13-14 April 2013. Meeting \# 1089. http://www.ams.org/amsmtgs/2210_abstracts/1089-03-60.pd $\mathrm{f}$

[9] P.Lindstrom, "First order predicate logic and generalized quantifiers",Theoria, Vol. 32, No.3. pp. 186-195, December 1966.

[10] F.R. Drake," Set Theory: An Introduction to Large Car-dinal",Studies in Logic and the Foundations of Mathe-matics,V.76.(1974).ISBN 0-444-105-35-2

[11] A.Kanamori, "The Higher Infinite: Large Cardinals in Set Theory from Their Beginnings", (2003) Spriger ISBN 3-540-00384-3

[12] A. Brovykin, "On order-types of models of arith-metic". Ph.D. thesis pp.109, University of Bir-mingham 2000.

[13] W.V.O.,Quine, "New Foundation for Mathematical Logic,"American Mathematical Monthly,vol.44, \{1937) pp.70-80.

[14] R.B.Jensen,"On the consistency of a slight (?) mo-Dification of Quine's'New Foundation,' "Synthese, vol.19 (1969),250-263.

[15] M.R. Holmes,'The axiom of anti-foundation in Jensen's 'New Foundations with ur-elements,'“ Bulletin de la SocieteMathematique de la Belgique, Series B

[16] M.R. Holmes, "Elementary Set Theory with a Uni-Versal Set,"vol.10 of Cahiers du Centre de Logi- que, UniversiteCatholiquede Louvain Lovain-la-Neuve, Belgium,1998.

[17] M.R. Holmes, "The Usual Model Construction for NFU Preserves Information," Notre Dame J. Formal Logic, Volume 53, Number 4 (2012), 571-580.

[18] A. Enayat, "Automorphisms, MahloCardinals,and NFU," AMS Special Session. Nonstandard mod- els of Arithmetic and Set Theory. January 15-16 2003,Baltimore, Maryland.

[19] R.M.Solovay,R.D.Arthan,J.Harrison, "The cosis-tency strengths of NFUB," Front for the arXiv, math/9707207

[20] E.Mendelson, "Introduction to Mathematical Logic" ISBN-10:0412808307,ISBN-13:978-0412808302 